\title{
La parole écrite: entre la loi et la marginalité
}

\section{The written word: between the law and marginality}

\author{
Ivete Lara Camargos Walty ${ }^{1}$
}

Submetido em 22 de dezembro de 2016 e aprovado em 9 de fevereiro de 2017.

Résumé: Dans une recherche sur la littérature produite par des prisonniers politiques et autres, j'ai toujours essayé de répondre aux questions suivantes:

- Comment la prison est-elle représentée dans les écrits de la contemporanéité à la première personne dans la littérature brésilienne et québécoise?

- Dans ces écrits, quelle relation y a-t-il entre l'institution prison et les autres institutions telles que: la Famille, l'École, l'Église, l’État, face à la question de la violence?

- Comment le corps se dessine-t-il dans l'écriture face à la violence?

- Comment subjectivité et collectivité se conjuguent-elles pour figurer les sujets narratifs en question?

- Comment esthétique, éthique et politique s'associent-elles dans l'écriture de la prison?

Dans cette étude, j'analyse les livres Memórias de um sobrevivente (Mémoires d'un survivant), de Luís Alberto Mendes, et Bienvenue dans mon cauchemar, de Marie Gagnon, en observant notamment le rôle de l'écriture en situation-limite entre la marginalité et la loi.

Mots-clés: Écriture. Prison. Corps. Subjectivation. Loi. Marginalité.

Abstract: In a research on the literature produced by political and common prisoners, I am trying to answer the following questions:

- How is prison drawn in the contemporary first-person narrator from Brazilian and Québécois literatures?

- How is prison, in these writings, associated to other institutions, i.e., family, school, church, state, in relation to the violence issue?

- How is the body drawn in these writings in its relation with violence?

- How are subjectivity and collectiveness conjugated in the figuration of the involved narrative subjects?

- How are ethics, aesthetics and politics conjugated in prison writings?

In this work, I analyze the books Memórias de um Sobrevivente, by Luís Alberto Mendes and Bienvenu dans mon cauchemar, de Marie Gagnon, observing especially the importance of writing in a limit situation between marginality and law.

Keywords: Writing. Prison. Body. Subjectivation. Law. Marginality.

Dans ma recherche sur la littérature produite par des prisonniers, politiques ou non, j’ai cherché à répondre aux questions suivantes:

- Comment la prison est-elle représentée dans les écrits de la contemporanéité à la première personne, dans la littérature brésilienne et québécoise? 
- Dans ces écrits, quelle relation y a-t-il entre l'institution prison et les autres institutions telles que: la Famille, l’École, l'Église, l'État, face à la question de la violence?

- Comment le corps se dessine-t-il dans l'écriture face à la violence?

- Comment subjectivité et collectivité se conjuguent-elles pour figurer les sujets narratifs en question?

- Comment esthétique, éthique et politique s'associent-elles dans l'écriture de la prison?

Dans ce travail j'analyse, en particulier, les livres Memórias de um sobrevivente (Mémoires d'un survivant), de Luís Alberto Mendes, et Bienvenue dans mon cauchemar et Lettres de prison, de Marie Gagnon, en observant le rôle de l'écriture en situationlimite entre la marginalité et la loi.

De la torture au corps abject.

Je suis un fils de pute Pas la peine de couper (rires). Je suis un fils de pute. Je suis un angoissé et ma seule satisfaction dans la vie, c'est d'écrire. Quand j'écris un beau texte, logique, auquel je crois et que j'aime, je me sens vraiment heureux. Il n'y a qu'à ce momentlà que je suis heureux (Luiz Alberto Mendes, entretien dans Livre Opinião. Ideias em debate).

Luiz Alberto Mendes, prisonnier brésilien depuis les années 1970, a écrit ses mémoires en prison et les a publiées au bout de 10 ans, alors qu'il était encore en prison, où il est resté pendant trente ans de plus. Constituant une trilogie, il a aussi publié Às cegas ('̀ l'aveuglette) et Memórias de um sobrevivente III (Mémoires d'un survivant III), aux prestigieuses éditions Companhia das Letras. Dans son premier livre, écrit en prison, il se penche sur ses expériences de vie, depuis son enfance dans une famille pauvre, marquée par la violence du père et la douceur de la mère. Le récit raconte, en spirale, le cercle vicieux de la violence subie puis exercée. Subie par lui à travers les raclées du père, les bagarres avec les autres prisonniers et les tortures pratiquées par les policiers. Exercée par lui dans des vols à la tire de pickpocket, dans le centre-ville de São Paulo ou des holdup de magasins et de stations-service, ainsi que lors des confrontations avec la police ou avec les autres prisonniers. Entre les hold-up, les détentions, entre les détentions, les holdup, du sexe, des drogues et du rock and roll. Dans ce mouvement circulaire où bandits 
et policiers changent de rôles, au fur et à mesure que ceux-ci sont exploités par ceux-là - qui sont presque toujours corrompus -, on exhibe le monde de derrière les barreaux et les cachots de la société de consommation autoritaire et intoxiquée. Ses institutions s'exposent en ruines: famille, école, prison, État, aucune d'elles n'accomplit la fonction à laquelle elle devrait, dans l'idéal, se consacrer.

L'auteur prend la forme de plusieurs énonciateurs : fils amoureux ou révolté, voleur récalcitrant, lecteur et écrivain critique. Qu'il soit violent et révolté, ou bien solidaire et juste, il montre la force de la loi du milieu, et poursuit une identité qui se gangrène comme son corps sinistré, presque supprimé:

J'ai pris des coups, encore et encore, j'ai pleuré, imploré pour qu'on me tue mais qu'on cesse de me battre. J'ai déféqué, uriné, involontairement, la pièce est devenue d'une puanteur extrême. Ils m'ont barbouillé la bouche de mes selles et ils me battaient, me battaient encore, sans arrêt, sans aucun répit. J'avais mal aux jambes, comme si le tuyau les pénétrait. Le choc me secouait tout entier et, avec le temps, cessa de se faire sentir.

$[\ldots]$

Durement torturé, je fus trainé jusqu'à la cellule, sentant la merde et la pisse (p. 336).

Le corps présenté de forme scatologique devient métonymie de la société mentionnée ici directement et indirectement:

\begin{abstract}
Nous étions conscients que ceux qui nous avaient suppliciés l'avaient fait au nom d'une société. Une société qui nous rejetait, nous brutalisait, nous éloignait, et qui nous détruisait presque. Et pire encore : une société qui avait besoin de ces monstruosités pour se maintenir. La torture était une institution sociale.

Si nous avions été dans un pays moins démagogique et plus civilisé, peut-être aurions-nous reçu la peine de mort. Nous serions probablement condamnés à mort. Et cela pourrait même être juste. Mais au nom de cette justice, nous aurions dû recevoir un traitement qui respectait les conditions existentielles humaines durant notre enfance et notre adolescence (p. 346).
\end{abstract}

Il est bon de rappeler qu'à l'époque où ce texte a été écrit, le Brésil était en pleine dictature civile militaire, instaurée en 1964, ce qui rend sa critique sociale et politique plus explicite, sans pour autant signifier que la question de la violence en prison et des 
problèmes sociaux aient été étouffés avec la fin de l'état d'exception. Le texte de Mendes expose le corps décomposé du prisonnier qui dénonce l'abjection du corps social.

\section{De la saleté à l'asepsie}

Une vie étrange m'envahit, une peine qui grandit, qui me noie, qui me tue. J'ai besoin d'aide. J'ai besoin d'aide. (Marie Gagnon, Bienvenue dans mon cauchemar)

De la violence physique des prisons brésiliennes on passe à la violence aseptisée des prisons canadiennes, décrite par Marie Gagnon dans les ouvrages Bienvenue dans mon cauchemar (1999) et Lettres de prison (2002). Les deux œuvres se réfèrent à deux prisons différentes: Tanguay (Montréal) et Joliette (Joliette - Québec). La première, fermée depuis, est décrite comme plus précaire et moins propre que la seconde, une prison fédérale. C'est pourtant dans cette dernière que Marie Gagnon constate une plus grande violence, l'asepsie de l'esprit:

Tanguay abritait des brutes véritables, mais aussi des filles qui se sacrifiaient pour d'autres. Des êtres colorés. Ici, c'est tout gris. Làbas, c'était dur, mais c'était vrai. J'y ai assisté à des scènes à se mettre à genoux et même à croire en Dieu. Ici, tout est tiède et faux. Tout est aseptisé. Geôle chrome, aussi froide qu'une morgue. Et tous ces psycolo-intervenants qui vont psalmodiant leurs morbides sentences (GAGNON, 2002, p. 136).

C'est l'auteure elle-même qui établit la relation entre cette prison et son pays: «Le Canada est un pays de pharisiens nantis, hypocrites et sournois. Joliette, c'est pareil.» (p.137). Réagissant aux mécanismes de la prison et à la classification des prisonniers, elle dit aussi qu'elle ne veut pas être la personne que la prison veut qu'elle soit:

La société m'a tourné le dos: elle m'a menottée, entravée, enfermée; elle a tripoté dans mon crâne en vaines tentatives d'en extirper l'original pour le remplacer par le commun. Eh bien! Moi aussi, je lui tourne le dos. Je fais 'Fi' de cette société qui m'enchaîne pour avoir la paix. Je me rends de plus en plus compte que je ne suis pas ici pour payer ma conduite passée, ni pour changer un comportement fautif, mais pour devenir comme les autres. Non, je me reprends: pas comme les autres, mais plutôt comme ce qu'il y a de plus médiocre chez les autres (GAGNON, 2002, p. 88).

Ou encore: 
On m'a cataloguée 'antisociale grave'. Je prends ça comme un compliment. Non que je veuille nuire aux autres: des actes illicites, je ne veux plus en commettre. Mais je ne veux pas ressembler à la citoyenne dont Joliette est le reflet. Elle est trop moche (GAGNON, 2002, p. 138).

Comment rapprocher deux personnes si différentes, de deux pays si divers? Le premier est pauvre, né dans la grande banlieue de São Paulo, presque analphabète. La seconde, titulaire d'une maîtrise en Lettres, est devenue une lectrice compétente et critique. Le corps du premier est massacré, affaibli, dilacéré, mais il réagit et survit à chaque séance de torture. La seconde réagit à ce qu'elle considère comme un modelage de cerveau et une asepsie de la personnalité. Tous deux sont victimes de deux types de violence, celles que Zizek nomme subjective et objective. La première, plus visible, serait celle qui est «exercée par des agents sociaux, des individus maléfiques, des appareils répressifs disciplinés et des foules fanatiques» (ZIZEK, 2014, p. 24). La seconde soutiendrait invisiblement «la normalité niveau zéro contre laquelle nous percevons la contrepartie d'une violence subjective.» (2014, p. 18). On associe, de cette manière, à la violence systémique celle qui est «inhérente à un système», «sous des formes plus subtiles de contrainte qui soutiennent les relations de domination et d'exploitation» (2014, p. 24). Tous deux appartiennent ainsi à ce que Foucault, dans Surveiller et punir (1975), nomme le corps docile, un type d'observatoire qui permet un meilleur contrôle et le maintien de l'ordre social, politique et économique.

En dehors de cela, un autre point fort les unit ; tous deux deviennent écrivains, bien que suivant des parcours différents. En racontant leur vie sous forme de biographie ou de lettres adressées à des proches, ils résistent par l'écriture, en se construisant en tant que sujets face à l'autre, y compris celui qui ne les connaît pas, mais les lit. Tous deux dépendent de la médiation d'un tiers; dans le cas de Mendes, Eneida, une étudiante en lettres qui lui écrivait, ainsi que par l'écrivain Fernando Bonassi et le médecin Dráuzio Varela, jouent un rôle important dans la reconnaissance de la capacité du nouvel écrivain, et dans la réalisation de sa publication.

J'ai parlé du livre qui dormait au fond d'un tiroir. Il a voulu le connaître. Plus par amitié, et pour avoir ainsi quelque chose à offrir au nouvel ami, j'ai fait venir le livre. Le gars a commencé 
à lire, et ses commentaires étaient très enthousiastes. Il l'aimait vraiment, et mettait mon texte en valeur. Ce n'était pas uniquement l'histoire, mais également le style qui lui plaisait bien. J'en ai été immensément content. Mais l'ami est allé plus loin : il voulait que le livre soit publié. Il m'a encouragé à faire une révision du texte, d'un bout à l'autre, à le mettre à jour, et il s'est engagé à chercher activement de l'aide pour le faire publier (MENDES, 2009, p. 411).

Marie Gagnon, pour sa part, correspond avec son éditeur, Jean-Yves Soucy, de VLB Éditeur, qu'elle appelle son ami et complice. Cet éditeur aussi lui demande une révision du texte avant publication: «Tu me demandes de corriger mes Lettres de prison. Je peux bien essayer, mais accorde-moi du temps. Rien n'avance rapidement ici, en ce lieu aseptisé... pas même ma plume» (p. 161). Dans une lettre du 23 avril 2001, elle lui narre la scène où un policier lui demande si elle est bien l'écrivaine qu'il a vu à la télévision: «Comment quelqu'un qui a ton talent peut-il vivre de vols et de vagabondage?» Ce à quoi elle répond, consciente de la relation entre le langage et l'expérience humaine:

- Merci pour le talent que vous me prêtez, monsieur, mais c'est justement sur la base de mon expérience d'itinérante et de criminelle que je compte réaliser mon œuvre littéraire, si tant est que le mot œuvre n'est pas ici déplacé (p. 82).

Dans les deux cas, il y a partenariat entre l'intellectuel et celui que se trouve derrière les barreaux, ce qui mène à un processus de lignes de fuite au sein de la répression. Pas seulement la répression proprement dite, mais l'impossibilité du partage de la parole.

Il est curieux de vérifier quelques exemples de la manière dont la parole circulait dans les cellules disciplinaires du pénitencier brésilien, par la tuyauterie des sanitaires:

Il m'apporta le message de Carlão disant que je tire la chaîne des sanitaires pour brancher le téléphone, il voulait me parler. Il expliqua que Carlão habitait en face et que la tuyauterie des wc était un canal d'appel, de communication. (p. 372)

$[\ldots]$

C'était le téléphone, notre fétide véhicule de communication. L'odeur était terrible, il fallait avoir de l'estomac. (p. 374)

Cette parole liée à la scatologie humaine cherche à réduire les distances, à l'intérieur et hors de la prison. De la même manière, la parole a du mal à circuler dans la 
prison aseptisée. Le mot «murs» est constamment répété dans les deux livres de Marie Gagnon, tout comme les murailles et les barreaux mentionnés par Mendes, auxquels s'ajoutent les termes qui se réfèrent à la couleur grise et au froid qui marquent le plus leur processus d'écriture:

J'ai besoin d'air. J'ai peur de m'incruster entre ces murs, de m'y fondre, qu'on me confonde avec eux. Ma peau deviendra-t-elle aussi froide que le ciment? Mon cœur, aussi dur? (GAGNON, 1997, p. 54).

À cette image, s'ajoute celle du manque d'air, de la difficulté à respirer, comme dans le poème «Murs»:

\author{
Fatiguée des murs \\ Partout des murs \\ Encore des murs \\ Même! Un plafond de murs \\ D'où suintent voix et cris \\ Et pire... des murmures \\ Pas de silence, que des bruits \\ J'étouffe, un soupir \\ Laissez-moi dormir! \\ (GAGNON, 1997, p. 65)
}

Ainsi, telle une forme de respiration, de recherche du souffle vital, la parole est peinte sur le mur de la prison et du corps, comme on peut le voir dans le poème «Mot» :

Elle chiale l'ombre épaisse et grotesque

Sur les murs de Tanguay, presque

D'une bave imbécile, une fresque.

(GAGNON, 1997, p. 61) 


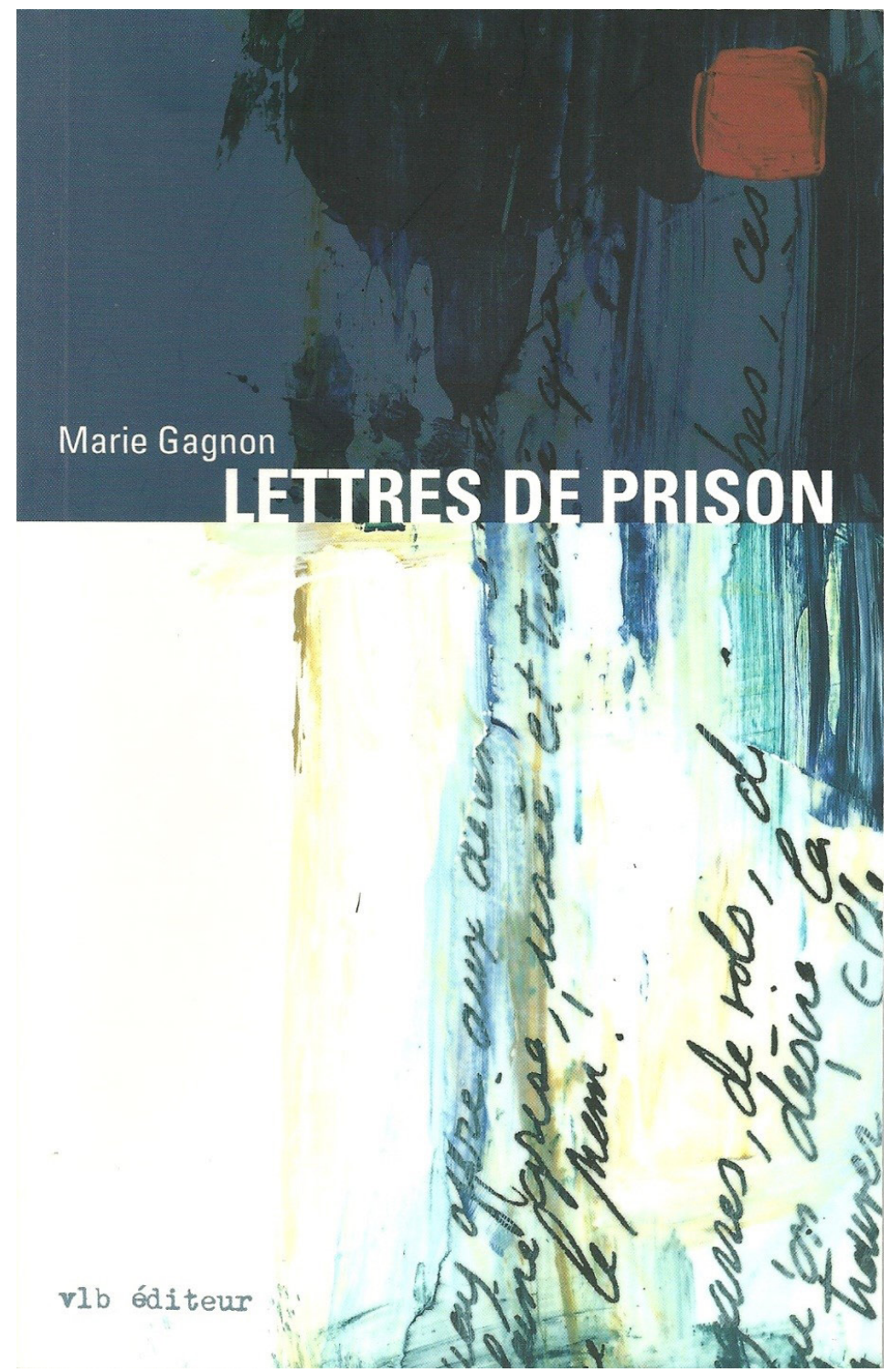

FIGURE: couverture du livre

Tout comme Mendes qui déclare que l'écriture l'a aidé à revivre les évènements et à réfléchir dessus, au point de devenir un besoin: - «L'intention est d'écrire toujours et pour toujours» (p. 409) -, Marie met son identité dans l'écriture: «Je ne me sens d'aucune 
ville, d'aucun pays, d'aucun groupe social. Ma contrée, c'est l'écriture; mon village, les mots $(2002$, p. 80$)$.

On peut pourtant se demander si cet exercice d'écriture en quête de liberté, de couleurs, de sons, comme une forme d'appartenance, est suffisant pour une recherche démocratique de construction identitaire. Avant de discourir sur ce point, il faut cependant relever, avec Zizek (2014), une autre forme de violence, qui est symbolique et se structure dans le langage lui-même. Comme le montre bien cet auteur, cela résulte du fait que le langage, bien qu'il soit le fondement de la condition humaine, ou pour cela même, n'est pas exempt de violence. En analysant le langage comme une condition de la non-violence postulée par d'autres auteurs, y compris Hannah Arendt, il affirme:

[...] le langage, moyen par excellence de la non-violence et de la reconnaissance mutuelle, implique une violence inconditionnelle. Autrement dit, c'est le langage lui-même qui induit notre désir à aller au-delà des limites convenables, en le transformant en un 'désir qui comporte l'infini', en l'élevant à une impulsion absolue qui ne pourra jamais être satisfaite (ZIZEK, 2014, p. 62).

Pour conclure sa réflexion à la lumière de la psychanalyse, Zizek prend position en se référant à ce qu'il appelle «'abîme du prochain»:

Bien qu'il puisse paraître qu'il existe ici une contradiction entre le mode selon lequel le discours constitue le noyau de l'identité du sujet et l'idée que ce noyau est un abîme insondable au-delà de la 'barrière du langage', il y a une solution simple à cet apparent paradoxe. La 'barrière du langage' qui me sépare à jamais de l'abîme de l'autre sujet est simultanément ce qui ouvre et maintient cet abîme - l'obstacle qui me sépare de l'Au-delà - et ce qui crée son image (ZIZEK, 2014, p. 67).

De toute façon, le langage serait l'espace-seuil dont parle Agamben $(2004,2007)$, car c'est en lui que l'homme expose sa fracture inconditionnelle, en se constituant comme sujet face à l'autre.

Ainsi, l'écriture raturée de Mendes et de Marie Gagnon montre bien plus que leur condition de prisonnier et que la prison comme métonymie de la société qui l'entretient, elle expose la condition de l'homme face à lui-même et face à l'Autre. 
Par conséquent, cela rappelle les propos de Rancière qui postule que «l'écriture est indissolublement deux choses en une: c'est le régime errant de la lettre orpheline dont aucun père ne garantit la légitimité, mais c'est encore la propre texture de la loi, inscription immuable de ce que la communauté a en commun.» (RANCIERE, 1995, p. 9)

La parole du prisonnier met doublement en scène cette condition de l'écriture. Et cela, parce que cette écriture ouvre le jeu entre dé-subjectivation et subjectivation, l'insistance à se voir agir sur le monde, dans un mouvement de la conscience qui marque l'être humain, surtout visible dans des situations-limites. Les paroles de Mendes, avec leur jeu d'articles défini et indéfini devant l'expression «être humain» sont révélatrices de cette situation:

Il ne s'agit pas ici d'une de ces histoires très édifiantes, bien au contraire, mais celui qui prendra la peine de la lire, et j'espère qu'ils seront nombreux, va sentir que c'est juste l'histoire d'un être humain. [...] Mais je crois que je suis parvenu à comprendre un peu de cette confusion, de cet apparent chaos et de la folie qu'est l'être humain (MENDES, 1999, p. 415 - souligné par nous).

Traduction de Janine Houard

\section{Références}

AGAMBEN, Giorgio. Estado de exceção: homo sacer II. Trad. Iraci D. Paleti. São Paulo: Boitempo, 2004.

AGAMBEN, Giorgio. Homo sacer: o poder soberano e a vida nua. Trad. Henrique Burigo. Belo Horizonte: Editora da UFMG, 2007.

ARENDT, Hannah. A condição humana. Trad. Roberto Raposo. Rio de Janeiro: Forense Universitária, 2001.

FOUCAULT, Michel. Surveiller et punir. Paris: Gallimard, 1975.

GAGNON, Marie. Bienvenue dans mon cauchemar. Montréal: vlb éditeur, 1997.

GAGNON, Marie. Lettres de prison. Montréal: vlb éditeur, 2002.

MENDES, Luiz Alberto. Memórias de um sobrevivente. São Paulo: Companhia das Letras, 2099.

RANCIÈRE, Jacques. A partilha do sensível: estética e politica. Trad. Mônica Costa Netto. São Paulo: Editora 34, 2009. 
RANCIÈRE, Jacques. Políticas da escrita. Trad. Raquel Ramalhete, Rio de Janeiro: Editora 34, 1995.

ZIZEK, Slavoj. Violência: seis reflexões laterais. Trad. Miguel Serras Pereira. São Paulo: Boitempo, 2014.

\section{Notes}

1 Professora adjunta da Pontifícia Universidade Católica de Minas Gerais, pesquisadora do CNPq, Belo Horizonte, MG, Brésil iwalty2@yahoo.com.br. 